\title{
Maus-tratos a idosos: revisão integrativa da literatura
}

\author{
Elderly maltreatment: integrative review of the literature \\ Abuso contra ancianos: revisión integradora de la literatura
}

\section{Annelissa Andrade Virgínio de Oliveira', Debora Raquel Soares Guedes Trigueiro', Maria das Graças Melo Fernandes", Antonia Oliveira Silva"I}

\author{
' Universidade Federal da Paraíba, Centro de Ciências da Saúde, \\ Programa de Pós-Graduação em Enfermagem (Mestranda). João Pessoa-PB, Brasil. \\ "Universidade Federal da Paraíba, Centro de Ciências da Saúde, Departamento de Enfermagem Clínica, \\ Programa de Pós-Graduação em Enfermagem. João Pessoa-PB, Brasil. \\ I' Universidade Federal da Paraíba, Centro de Ciências da Saúde, Departamento de Enfermagem de Saúde Pública e \\ Psiquiatria, Programa de Pós-Graduação em Enfermagem. João Pessoa-PB, Brasil.
}

\author{
Submissão: 14-04-2011 Aprovação: 22-01-2013
}

\section{RESUMO}

O estudo objetivou identificar, a partir de uma revisão integrativa da literatura, o conhecimento científico produzido entre 2005 e 2009, sobre maus-tratos contra idosos. Para tal, foram selecionados dezesseis estudos nos bancos LILACS e SciELO e na base BDENF. Dentre os dezesseis estudos, 56,25\% compreendiam artigos originais. O periódico que mais publicou foi o Caderno de Saúde Pública (25\%), sendo a maioria dos estudos desenvolvidos no Sudeste (56,25\%). O levantamento revelou que a violência física foi predominante entre os idosos, sendo o domicílio o ambiente onde frequentemente ocorre a agressão, sendo os familiares os principais agressores. Entre os idosos agredidos, observou-se que as mulheres são as vítimas mais acometidas. Esses achados suscitam o desenvolvimento de outras pesquisas que possam clarificar as múltiplas dimensões da violência contra o idoso.

Descritores: Enfermagem; Enfermagem Geriátrica; Maus-Tratos ao Idoso; Idoso.

\section{ABSTRACT}

The study aimed to identify, through an integrative literature review, scientific knowledge, produced between 2005 and 2009 about the mistreatment of the elderly. It was selected sixteen studies in the data banks LILACS, SciELO and BDENF. Of the sixteen studies, 56.25\% comprised original articles. The journal that published the most was the Public Health Journal (25\%), being the majority of the studies from the Southeast $(56.25 \%)$. The survey revealed that the physical violence was prevalent among the elderly; the home was the environment where aggression occurs more frequently; and family members were the main aggressors. Among the elderly attacked, it was observed that women are the victims most affected. These findings raise the development of other researches that might clarify the multiple dimensions of violence against the elderly.

Key words: Nursing; Geriatric Nursing; Elder Abuse; Aged.

\section{RESUMEN}

El estudio tuvo como objetivo identificar, a través de una revisión bibliográfica integrada, los conocimientos científicos, producidos entre 2005 y 2009 sobre maltrato a ancianos. Con este fin, se seleccionaron dieciséis estudios en los bancos LILACS e SciELO e en la base de datos BDENF. De ellos, 56,25\% eran artículos originales. La revista más utilizada para publicación, fue el Cuaderno de Salud Pública (25\%), y la mayoría de los estudios se desarrollaron en el Sudeste (56,25\%). La encuesta reveló que la violencia física era la más frecuente entre los ancianos; el entorno familiar es adonde ocurre con mayor frecuencia la agresión; y los familiares son los principales agresores. Entre las personas de edad que fueron atacados, se observó que las mujeres son las víctimas más afectadas. Estos resultados plantean el desarrollo de otras investigaciones que puedan aclarar las múltiples dimensiones de la violencia contra los ancianos.

Palabras clave: Enfermería; Enfermería Geriátrica; Maltrato al Anciano; Anciano. 


\section{INTRODUÇÃO}

Nas últimas décadas, o Brasil tem experimentado o aumento da expectativa de vida em que a realidade do envelhecimento da população tornou-se um dos principais desafios da modernidade ${ }^{(1)}$. O cenário se torna mais agravante quando, somado as desigualdades sociais, tem-se a falta de informações, o preconceito e o desrespeito à pessoa idosa. A velhice carrega os estigmas da incapacidade funcional e social do indivíduo, reduzindo o idoso, muitas vezes, a um fardo para os seus responsáveis, concorrendo assim, à exclusão familiar e social.

Aliados a esses fatores, o idoso carrega a bagagem da sua própria construção sociocultural acerca do avançar da idade. Sentimentos como medo da morte, inutilidade, solidão, desprezo e outros sofrimentos podem perpassar suas emoções, tornando a trajetória ainda mais dolorosa. É preciso dar conta desta incômoda realidade em que os próprios mitos do enveIhecimento já conformam um tipo de violência (a simbólica), pois retratam uma cobrança de eterna juventude, além de preconceitos que impedem investimentos da sociedade para o bem-estar na velhice.

Diante das possíveis dificuldades enfrentadas pelos idosos frente ao processo de envelhecer, a violência tem se mantido no quadro de preocupação mundial como um dos principais alertas à saúde pública, apontados em estudos que indicaram uma elevada prevalência da violência contra os idosos, se comparado a outros problemas de saúde com destaque epidemiológico ${ }^{(2-3)}$.

A Organização Mundial de Saúde (OMS) define violência contra o idoso como um ato único ou repetitivo ou mesmo a omissão, podendo ser tanto intencional como involuntária, que cause dano, sofrimento ou angústia(4). A mesma pode ser praticada dentro ou fora do ambiente doméstico por algum membro da família ou ainda por pessoas que exerçam uma relação de poder sobre a pessoa idosa.

A OMS, mediante o consenso internacional envolvendo todos os países participantes da Rede Internacional de Prevenção contra Maus-Tratos em Idosos, elencou sete tipos de violências: abuso físico ou maus-tratos físicos (reporta-se ao uso de força física); abuso ou maus-tratos psicológicos (envolve agressões verbais ou gestuais); a negligência (recusa, omissão ou fracasso por parte do responsável no cuidado com a vítima); a autonegligência (negação ou fracasso de prover a si mesma de cuidado adequado); o abandono (ausência, por parte do responsável, de assistência necessária ao idoso, a quem caberia prover custódia física e cuidado); abuso financeiro (exploração imprópria ou ilegal e/ou uso não consentido dos recursos financeiros de um idoso), e o abuso sexual (ato ou jogo sexual, destinado a estimular a vítima ou utilizá-la para obter excitação sexual e práticas erótico-sexuais ${ }^{(4)}$.

Além dessa categorização dos atos de violência contra a pessoa idosa, observa-se também uma classificação desses eventos em violência institucional ou estrutural, doméstica (intrafamiliar/interpessoal) e simbólica. Desse modo, é difícil identificar a violência exercida contra o idoso, uma vez que não se trata apenas de agressão física - perceptível especialmente pelas marcas corpóreas resultantes; mas, também, danos sociais, psicológicos e morais. Independente do tipo de abuso, seguramente resultará em sofrimento desnecessário, lesão ou dor, perda ou violação dos direitos humanos e redução na qualidade de vida do idoso(5).

Apesar da violência contra a pessoa idosa constituir-se um importante problema de saúde pública com visibilidade na contemporaneidade, o conhecimento científico produzido relativo à temática, especialmente no Brasil, ainda é escasso. Considerando esse fato, bem como a importância de se produzir novas reflexões que auxiliem na luta contra os maus-tratos aos idosos em diferentes dimensões, delimitou-se para este estudo a seguinte questão norteadora: Que dimensões da violência são abordadas no conhecimento científico produzido no Brasil no período de 2005 a 2009 no que concerne aos maus-tratos aos idosos? Para responder tal questão, este estudo teve como objetivo o de identificar, a partir de uma revisão integrativa da literatura, o conhecimento científico produzido no Brasil entre 2005 e 2009 sobre maus-tratos contra idosos.

\section{METODOLOGIA}

Trata-se de um estudo de abordagem qualitativa em que se optou pelo método da revisão integrativa para alcance do objetivo proposto. Este possibilita a síntese do estado da arte do conhecimento de um determinado assunto, apontando lacunas do conhecimento que precisam ser preenchidas com a realização de novos estudos como suporte à tomada de decisão e à melhoria da prática clínica, além de permitir a realização de uma síntese de múltiplos estudos publicados, viabilizando conclusões gerais a respeito de uma particular área de estudo ${ }^{(6-7)}$.

Uma revisão integrativa exige os mesmos padrões de rigor, clareza e replicação utilizada nos estudos primários ${ }^{(8)}$. Considerando isso, na operacionalização dessa revisão, foram percorridas as seguintes etapas: delimitação da questão de pesquisa (já apresentada); estabelecimento dos critérios de inclusão/exclusão para a seleção dos estudos a serem analisados; definição das informações a serem extraídas dos estudos selecionados; avaliação dos estudos incluídos na revisão integrativa; análise dos dados, interpretação dos resultados e apresentação da síntese da revisão ${ }^{(9-10)}$.

O levantamento bibliográfico foi realizado pela Internet, por meio da Biblioteca Virtual em Saúde (BVS), nas bases de dados LILACS (Literatura Latino-Americana em Ciências de Saúde), SciELO (Scientific Electronic Library Online) e na BDENF (Base de Dados Bibliográficos Especializada na Área de Enfermagem do Brasil). Para o levantamento dos artigos, utilizou-se o descritor "maus-tratos ao idoso", combinado com os termos "enfermagem" e "enfermagem geriátrica", utilizados para o refinamento da amostra.

Os critérios utilizados para a seleção da amostra foram: artigos que abordassem a temática em questão, escritos na língua portuguesa, abrangendo as diferentes áreas do conhecimento, publicados entre os anos de 2005 e 2009, em periódicos indexados nas bases de dados LILACS, SciELO e BDENF que tinham o texto completo disponibilizado on-line. 
Considerando esses critérios, foram identificados oitenta artigos nas bases de dados LILACS e SciELO. Na BDENF não havia nenhum estudo sobre a temática. Vale ressaltar que, após a leitura aprofundada desses artigos, foram excluídos 64 deles, por não atenderem aos critérios de inclusão. Dessa forma, a amostra final foi composta por dezesseis trabalhos científicos.

Para a obtenção das informações que respondiam a questão norteadora da pesquisa elaborou-se um formulário que contemplava a identificação do artigo e dos autores, fonte de localização, cenário geográfico em que foi desenvolvido, objetivos, delineamento e características do estudo, resultados, conclusões e recomendações. Para a análise dos dados foi utilizada a estatística descritiva seguida da interpretação dos achados, fundamentada na literatura pertinente.

\section{RESULTADO}

$\mathrm{Na}$ análise aqui empreendida, foram verificados os resultados apresentados nos tópicos que se seguem, os quais compreendem as características dos estudos, assim como os dados relacionados com os maus-tratos aos idosos contemplados nos artigos analisados.

Tabela 1 - Distribuição dos artigos focalizando maus-tratos aos idosos, publicados entre 2005 e 2009, conforme periódico. Brasil, 2010

\begin{tabular}{lc}
\hline Periódico & N (\%) \\
\hline Caderno de Saúde Pública & $4(25 \%)$ \\
Revista Eletrônica de Enfermagem & $2(12,5 \%)$ \\
Revista Latino-Americana de Enfermagem & $1(6,5 \%)$ \\
Revista Brasileira de Enfermagem & $1(6,5 \%)$ \\
Revista. Brasileira de Geriatria e Gerontologia & $1(6,5 \%)$ \\
Revista Brasileira de Psiquiatria & $1(6,5 \%)$ \\
Revista Brasileira de Saúde Materno-Infantil & $1(6,5 \%)$ \\
Revista Saúde Pública & $1(6,5 \%)$ \\
Interface - Comunicação, Saúde, Educação & $1(6,5 \%)$ \\
Psicologia: Revista da Vetor Editora & $1(6,5 \%)$ \\
Saúde e Sociedade & $1(6,5 \%)$ \\
São Paulo em Perspectiva & $1(6,5 \%)$ \\
\hline
\end{tabular}

Fonte: Dados da pesquisa. 2010.

Quanto aos periódicos que mais publicaram sobre o assunto, destacaram-se o Caderno de Saúde Pública (25\%) e a Revista Eletrônica de Enfermagem (12,5\%). No concernente ao período de publicação, 2008 foi o ano em que houve maior número de publicações sobre o tema (43,75\%), seguido, consecutivamente, de 2007 (31,25\%) e de 2006 (18,75\%). Não foram verificados artigos publicados nos anos 2005 e 2009.

A maioria dos estudos foi desenvolvida na região Sudeste, com destaque para os estados do Rio de Janeiro $(31,25 \%)$ e São Paulo (25\%). Faz-se necessário refletir que esta região concentra a maior quantidade de Programas de Pós-Graduação e de pesquisadores do nosso País, fato que justifica a maior quantidade de produções. Entretanto, destaca-se a considerável quantidade de produções da região Nordeste (25\%), demonstrando o avanço dessa região na pesquisa científica. Ressalta-se, ainda, a ausência de pesquisas realizadas na região Norte do país.

Com relação ao tipo de estudo utilizado pelos pesquisadores para abordar a temática, quatro estudos eram exploratório-descritivos; os demais tipos de estudo, estão descritos na tabela 2.

Tabela 2 - Distribuição dos artigos focalizando maus-tratos aos idosos, conforme tipo de estudo. Brasil, 2010

\begin{tabular}{lc}
\hline Tipo de estudo & N (\%) \\
\hline Estudo descritivo & $4(25 \%)$ \\
Estudo exploratório-descritivo & $4(25 \%)$ \\
Revisão sistemática da literatura & $2(12,5 \%)$ \\
Estudo retrospectivo documental & $1(6,25 \%)$ \\
Artigo de atualização & $1(6,25 \%)$ \\
Inquérito de base populacional & $1(6,25 \%)$ \\
Não informado & $3(18,75 \%)$ \\
\hline
\end{tabular}

Fonte: Dados da pesquisa, 2010

Quanto às formas de maus-tratos aos idosos expressas nos trabalhos analisados, a violência física foi destacada mais frequentemente, tendo índices de prevalência bastante variáveis, dentre eles: $2 \%$ a $10 \%{ }^{(11)} ; 3,2 \%^{(12)} ; 10,1 \%^{(13)} 16 \%^{(14)}$; sendo o maior percentual de $31,8 \%{ }^{(15)}$. No tocante aos demais tipos de violência, a de natureza psicológica, a exemplo da "falta de respeito"(16), agressões verbais, insultos e outros rituais; assim como a negligência e o abuso financeiro também foram analisadas pelos autores de forma significativa ${ }^{(14-15,17-18)}$. Chama a atenção também a violência implícita que acomete os idosos de maneira velada, quando estes são desestimulados a realizar ações para as quais têm capacidade cognitiva e emocional apropriadas $^{(16)}$.

Os textos apresentam consenso quanto ao cenário físico, ou lócus da violência. Nesse sentido, o domicílio é apresentado pelas pesquisas como o ambiente onde mais frequentemente ocorrem as diferentes formas de agressão aos ido$\operatorname{sos}^{(12-13,15)}$. Salienta-se que, nas famílias, os idosos podem ter, segundo o grau de parentesco, até sete agressores (cônjuges, filhos, netos, genros, noras, irmãos, sobrinhos) $)^{(12,14-15,19)}$. 
Quanto ao perfil do idoso agredido, um estudo de revisão sistemática(20) identificou várias pesquisas que salientaram diferenças na prevalência de maus-tratos entre os sexos, apontando que as mulheres foram mais agredidas que os homens ${ }^{(5,15)}$, especialmente aquelas com oitenta e mais anos, deprimidas, confusas ou extremamente fragilizadas.

Dos dezesseis artigos que atenderam aos critérios adotados para este estudo, três $(18,75 \%)$ tinham como objetivos realizarem adaptação de instrumentos de rastreamento e avaliação de violência no idoso ${ }^{(21-22-23)}$; destes, dois estudos descreviam as fases de adaptação transcultural para o português de instrumentos utilizados em outros países para a identificação de risco de violência doméstica em idosos ${ }^{(21)}$ e para suspeição de violência contra o idoso ${ }^{(22)}$, e um estudo que fez a revisão dos instrumentos de rastreamento e avaliação de violência no idoso ${ }^{(23)}$.

\section{DISCUSSÃO}

$\mathrm{Na}$ análise dos resultados ora descritos, verificou-se que, apesar da existência de periódicos específicos sobre a temática, a exemplo da Revista Brasileira de Geriatria e Gerontologia, a maioria dos artigos foi publicada em periódicos gerais, o que dificulta a rápida atualização do conhecimento. Além disso, a inexistência de um periódico de enfermagem no Brasil voltado para a Gerontologia desfavorece a produção de pesquisas na área e, de algum modo, dificulta diretamente a divulgação do conhecimento produzido pelos enfermeiros.

Sendo a BDENF a base que publica estudos da área de Enfermagem, é preocupante o fato de a mesma não apresentar referências relativas a um tema tão relevante como maus-tratos à pessoa idosa. Alguns dos estudos localizados em outras bases de dados são divulgados por meio de periódicos da Enfermagem. Tal realidade suscita o seguinte questionamento: Por que a base de dados BDENF apresenta lacuna de informações no tocante à temática em análise? Torna-se imperativo a busca de resposta para esta questão.

Outro ponto importante a ser refletido é o sistema de busca do banco de dados SciELO, uma vez que na pesquisa realizada só foram identificadas sete referências. Entretanto, a mesma pesquisa, feita por outros meios, tais como o site Google, identificou um número maior de referências disponíveis na referida base de dados.

Dentro do intervalo de tempo delimitado para a amostra, observa-se um aumento progressivo do número de publicações entre os anos de 2005 a 2008, com significativo decréscimo no ano de 2009. Assim sendo, questiona-se: esse decréscimo acentuado estaria relacionado à diminuição do interesse dos pesquisadores pela temática ou à dificuldade de publicar artigos de revisão na área? Ressalta-se, portanto, a necessidade de maiores investimentos em pesquisas nesse campo a fim de impedir que o grande contingente de idosos continue sofrendo de violência de forma silenciosa.

Apesar da problemática dos maus-tratos aos idosos ser antiga, observa-se que a questão da violência contra os idosos ainda é pouco considerada pela sociedade em geral(24). É imprescindível, então, que os saberes produzidos levem a população a se sensibilizar frente aos diferentes tipos de violências na própria comunidade e a intervir nessas ocorrências denunciando imediatamente aos órgãos jurídicos competentes para as providências cabíveis.

A temática da violência não envolve só o idoso penalizado pelos maus-tratos, mas, também, seus familiares e a comunidade de abrangência. No âmbito social, deve haver uma participação expressiva dos profissionais de saúde no cuidado à vítima, de forma articulada e interdisciplinar com outros setores sociais, a fim de proteger a pessoa idosa e punir os responsáveis. Neste sentido, também é competência do sistema de saúde contribuir para a reversão dos elevados níveis de mortalidade provenientes desse agravo e suas consequências: medo, alienação, estresse pós-traumático ou mesmo a depressão, na vigilância e na criação de condições para que esse tipo de violência não aconteça.

Entre outros sentimentos que podem, inevitavelmente, ser expressos pelos idosos, ainda se destacam o temor da retaliação ou represália especialmente no âmbito familiar; a culpa de gerar um conflito; a vergonha da situação; e o medo de ser internado em um asilo. A vivência com os agressores, por sua vez, pode não só afetar a saúde do idoso, como constituir um dos grandes empecilhos para que a vítima denuncie.

Logo, ressalta-se que tanto os profissionais que atuam na rede básica de saúde, quanto àqueles que prestam ações nos serviços de emergência precisam de capacitação específica para que possam identificar e avaliar casos de violência. Durante a assistência, devem observar os sinais e as marcas deixadas por lesões e traumas em idosos que comparecem aos serviços e não conseguem mais sair com vida ${ }^{(25)}$.

Dados da OMS e do INPEA- International Network for the Prevention of Elder Abuse ${ }^{(4)}$ indicam que a ocorrência de violência contra os idosos no Brasil, a partir de relatos fornecidos sobre o processo de envelhecer, relaciona-se ao sentimento de "fossilização" que vivenciam quando são privados da participação social, inutilizados pela aposentadoria e marginalizados pelos seus familiares.

Além da desvalorização moral, outros tipos de maus-tratos são apontados nas demais investigações realizadas como: a negligência, o abandono, a violência psicológica por meio de insultos e ameaças, apropriação indevida de bens materiais e agressões de ordem física ${ }^{(5,11,13,17,26)}$. Observa-se ainda a falta de indicadores pertinentes ao abuso sexual como forma de violência contra os idosos.

Os principais perpetradores de maus-tratos contra os idosos, por sua vez, são os próprios familiares da vítima, levando-se em consideração o grau de dependência que se estabelece. Depender de outra pessoa significa estar em poder dela, tornando a oportunidade de coerção ainda maior. Vale ressaltar que o risco de abuso ou violência física é maior para os idosos que vivem com familiares devido à maior oportunidade de contato interpessoal, gerando conflitos e tensões ${ }^{(11)}$. Além disso, as características socioculturais da população idosa brasileira apontam o risco de situações de sobrecarga dos familiares de idosos dependentes como um importante determinante para situações de abusos, negligência e maus-tratos, caracterizados como elementos constitutivos da violência intrafamiliar. 
Doenças crônicas não transmissíveis, limitações motoras, deficiências cognitivas ou perda do cônjuge são situações que podem fazer com que o idoso dependa de um membro da família. A mudança para uma nova residência acaba alterando a dinâmica da estrutura familiar que, em muitos casos, não consegue se adaptar às demandas de cuidado que uma pessoa idosa apresenta.

Essa dificuldade em adequar-se à figura de cuidador, que exige do familiar uma transformação no estilo de vida, aliada ao baixo recurso financeiro, intolerância, incompreensão e indisposição, pode gerar conflitos difíceis de serem administrados. Assim, o limite entre a dificuldade de cuidar do idoso e a violência é tênue, principalmente se forem considerados os maus-tratos psicológicos e a negligência ${ }^{(26)}$.

Além do exposto, ressalta-se que é no cenário da família onde mais veementemente os valores "arcaicos", condicionados historicamente e cristalizados pelas pessoas mais velhas, são questionados pelos mais jovens, ameaçando assim as relações de poder outrora estabelecidas e, consequentemente, gerando conflitos intergeracionais.

Quanto ao sexo, verifica-se maior vulnerabilidade da muIher idosa à violência, em especial, aquelas que já sofriam violência doméstica em idade adulta ${ }^{(27)}$. Esse dado, de algum modo, relaciona-se à violência de gênero, que revela uma cultura de discriminação contra a mulher ${ }^{(26)}$. Apesar da maior expectativa de vida representar maior risco de incapacidade e, por sua vez, de violência para as mulheres, o fato de a idosa ter sido agredida quando jovem parece exercer maior influência na ocorrência de maus-tratos.

Em relação à detecção de casos de maus-tratos, há considerável número de estudos (15\%) sobre adaptação de instrumentos de rastreamento e avaliação de violência no idoso, demonstrando a preocupação dos pesquisadores em ter meios fidedignos para rastrear, identificar, avaliar e, principalmente, intervir em situações de maus-tratos aos idosos. Entre estes instrumentos destacaram-se o Hwalek-Sengstock Elder Abuse Screening Test (H-S/EAST) e o Caregiver Abuse Screen $(\mathrm{CASE})^{(22-23)}$. Estudo realizado no Brasil ${ }^{(21)} \mathrm{fez}$ a revisão de instrumentos de rastreamento e avaliação de violência no idoso e apontou lacunas importantes nos processos de concepção, validação e adaptação desses instrumentos, ficando visível a necessidade de aprofundamento de pesquisas na área, especialmente aquelas voltadas à análise criteriosa do conceito, 0 que inclui os atributos definidores do problema.

Do mesmo modo, o amparo jurídico a esses idosos foi um aspecto destacado. Embora passível de análise e de aperfeiçoamento, a criação do Estatuto do Idoso foi uma das mais importantes conquistas desse grupo, tendo a finalidade de assegurar os direitos às pessoas com idade igual ou superior a sessenta anos, dever não apenas do Estado, mas, também, da sociedade e da família ${ }^{(28)}$.

Apesar da elaboração de leis que garantem a defesa da dignidade e do bem-estar na velhice, muitos esforços ainda precisarão ser desenvolvidos contra a violência à pessoa idosa, tendo em vista que, na maioria dos casos, o agressor é um familiar que se configura como a única pessoa que pode desempenhar o papel de cuidador, sendo angustiante e preocupante para o idoso denunciá-lo, bem como para os demais membros familiares, que não querem/podem se responsabilizar pelo idoso ${ }^{(26)}$.

Faz-se, portanto, indispensável repensar os vínculos familiares na perspectiva de tornar a sociedade apta a acolher seus idosos, de forma que tenham consciência de que o seu futuro também reservará as vicissitudes da velhice.

\section{CONSIDERAÇÕES FINAIS}

Os achados do estudo em questão apontam significativa prevalência de maus-tratos aos idosos, ressaltando-se, também, que essa prevalência não expressa a magnitude do problema. Observa-se elevada subnotificação, em virtude do caráter velado dessa forma de violência, especialmente dos casos ocorridos no cenário familiar, contexto em que se verificam os maiores conflitos intergeracionais. A maior parte dos cuidadores informais de idosos fragilizados ou incapacitados física e cognitivamente vivenciam situação de sobrecarga do cuidado, importante fator de risco de violência doméstica que pode envolver esses idosos.

Quanto aos tipos de maus-tratos, verificaram-se os de natureza psicológica, seguido da negligência. No entanto, chama-se atenção da sua inter-relação com outros tipos de violência, pois, em sua maioria, se sobrepõem. No tocante às características do idoso envolvido mais frequentemente na experiência em questão, destacaram-se as mulheres, particularmente as de maior faixa etária e mais fragilizadas. $\mathrm{O}$ agressor constitui, em especial, as pessoas que têm vínculo consanguíneo ou coabitam com o idoso.

Embora este estudo apresente limitações do ponto de vista do número de artigos analisados e do período (2005 a 2009) em que estes foram produzidos, seus achados asseguram que os maus-tratos a idosos são mais comuns do que se imagina e a problemática exige que se saia do campo imaginário para intervir no real, tanto no meio clínico como no contexto social. Isso requer políticas públicas e ações de saúde que expressem um compromisso maior com a ética e a defesa aos direitos humanos, contemplando todas as faixas etárias, sem desmerecer as marginalizadas pela sociedade.

Considerando essa perspectiva salienta-se que o setor saúde tem papel preponderante nesta missão. Cabe aos profissionais da área o empenho em prevenir, identificar, diagnosticar e oferecer os cuidados necessários aos idosos vítimas de violência, independente da natureza, bem como informar as autoridades competentes, a fim do desenrolar das medidas cabíveis para que o idoso não necessite voltar ao lócus da violência, reiniciando um novo ciclo.

Dada a importância da prevenção como parte resolutiva do problema da violência contra os idosos, ressalta-se a relevância da conscientização e da capacitação da sociedade civil, especialmente da população mais jovem, no sentido de romper com os preconceitos e respeitar a dignidade do cidadão idoso. Por fim, ressalta-se que ignorar a violência contra os idosos resulta num lamentável futuro para o envelhecimento mundial, considerando, em especial, a perspectiva da dignidade da vida humana. 


\section{REFERÊNCIAS}

1. Romero AD, Silva MJ, Silva ARV, Freitas RWJF, Damasceno MMC. Características de uma população de idosos hipertensos atendida numa Unidade de Saúde da Família. Rev Rene 2010;11(2):72-78.

2. Krug EG, Dahlberg LL, Mercy JA, Zwi AB, Lozano R. World report on violence and health. Geneva: World Health Organization; 2002.

3. Ministério da Saúde. Política nacional de redução de morbimortalidade por acidentes e violência. Brasília, DF: O Ministério; 2002.

4. World Health Organization. Missing voices: views of older persons on elder abuse. Geneva: World Health Organization; 2002.

5. Leite MT, Hildebrandt LM, Santos AM. Maus-tratos a idosos no domicílio: concepção de familiares. Rev Bras Geriat Gerontol 2008;11(2):209-21.

6. Benefield LE. Implementing evidence-based practice in home care. Home Health Nurse 2003;21(12): 804-11.

7. Polit DF, Beck CT. Using research in evidence-based nursing practice. In: Polit DF, Beck CT, editors. Essentials of nursing research. Methods, appraisal and utilization. Philadelphia : Lippincott Williams \& Wilkins; 2006. p. 457-94.

8. Beyea SC, Nicoll ELH. Writing an integrative review. Aorn J 1998;67(4):877-80.

9. Ganong LH. Integrative reviews of nursing research. Res Nurs Health 1987;10(1):1-11.

10. Mendes KDS, Silveira RCCP, Galvão CM. Revisão integrativa: método de pesquisa para a incorporação de evidências na saúde e na enfermagem. Texto Contexto Enferm 2008;17(4):758-64.

11. Laks J, Werner J, Miranda-Sá Júnior LS. Psiquiatria forense e direitos humanos nos pólos da vida: crianças, adolescentes e idosos. Rev Bras Psiquiatr 2006;28(2):80-5.

12. Gaioli CCLO, Rodrigues RAP. Ocorrência de maus-tratos em idosos no domicílio. Rev Latino-Am Enferm 2008;16(3):465-70.

13. Moraes $\mathrm{CL}$, Apratto Júnior $\mathrm{PC}$, Reichenheim ME. Rompendo o silêncio e suas barreiras: um inquérito domiciliar sobre a violência doméstica contra idosos em área de abrangência do Programa Médico de Família de Niterói, Rio de Janeiro, Brasil. Cad Saúde Pública 2008;24(10):2289-300.

14. Souza JA, Freitas MC, Queiroz TA. Violência contra os idosos: análise documental. Rev Bras Enferm 2007;60(3): 268-72.
15. Melo VL, Cunha JOC, Falbo Neto GH. Maus-tratos contra idosos no município de Camaragibe, Pernambuco. Rev Bras Saúde Mater Infant 2006;6(1):43-8.

16. Silva MJ, Oliveira TM, Joventino ES, Moraes GLA. A violência na vida cotidiana do idoso: um olhar de quem a vivencia. Rev Eletr Enf [periódico na internet]. 2008 [acesso em 15 jun 2010];10(1):124-36. Disponível em: www.fen. ufg.br/revista/v10/n1/pdf/v10n1a11.pdf.

17. Porto I, Koller SH. Violência contra idosos institucionalizados. Psic Rev Psicol Vetor Ed 2008;9(1):1-9.

18. Kullok AT, Santos ICB. As representações sociais de funcionários de uma Instituição de Longa Permanência sobre violência no interior de Minas Gerais. Interface Comun Saúde Educ 2009;13(28): 201-12.

19. Debert GG, Oliveira AM. A polícia e as formas de feminização da violência contra o idoso. Perspect 2007;21(2):15-28.

20. Espindola CR, Blay SL. Prevalência de maus-tratos na terceira idade: revisão sistemática. Rev Saúde Pública 2007;41(2):301-6.

21. Paixão Júnior $C M$, Reichenheim ME. Uma revisão sobre instrumentos de rastreamento de violência doméstica contra o idoso. Cad Saúde Pública 2006;22(6):1137-49.

22. Paixao CM, Reichenheim ME, Moraes $\mathrm{CL}$, Coutinho ES, Veras RP. Adaptação transcultural para o Brasil do instrumento Caregiver Abuse Screen (CASE) para detecção de violência de cuidadores contra idosos. Cad Saúde Pública 2007;23(9):2013-22.

23. Reichenheim ME, Paixão Júnior CM, Moraes CL. Adaptação transcultural para o português (Brasil) do instrumento Hwalek-Sengstock Elder Abuse Screening Test (H-S/EAST) utilizado para identificar risco de violência contra o idoso. Cad Saúde Pública 2008;24(8):1801-13.

24. Florêncio MVDL, Ferreira FMO, Sá, LD. A violência contra o idoso: dimensão ética e política de uma problemática em ascensão. Rev Eletr Enf [periódico na internet]. 2007[acesso em 18 jun 2010];9(3):847-57. Disponível em: www.fen.ufg.br/revista/v9/n3/pdf/v9n3a23.pdf.

25. Minayo MCS. Violência contra idosos: relevância para um velho problema. Cad Saúde Pública 2003;19(3):783-91.

26. Sanches APRA, Lebrão ML, Duarte YAO. Violência contra idosos: uma questão nova? Saude Soc 2008;17(3):90-100.

27. Phillips LR. Domestic violence and aging women. Geriatric Nursing 2000;21(4):188-95.

28. Ministério da Saúde. Estatuto do idoso. Brasília, DF: O Ministério; 2003. 